SLAC-PUB-12148

BABAR-PROC-06/094

\title{
Measurement of Direct CP Asymmetries in Charmless Hadronic B Decays
}

\author{
Emanuele Di Marco \\ Università di Roma "La Sapienza" \\ Physics Department, P.le Aldo Moro 2, 00185 Roma, Italy \\ (representing the BABAR Collaboration) \\ February 8, 2020
}

\begin{abstract}
We present recent results on time integrated and time dependent $C P$ violation for charmless hadronic $B$ decays using BABAR detector at the PEP-II B-factory.
\end{abstract}

Submitted to the the Proceedings of $33^{\text {rd }}$ International Conference on High-Energy Physics, ICHEP 06,

26 July_-2 August 2006, Moscow, Russia.

Stanford Linear Accelerator Center, Stanford University, Stanford, CA 94309

Work supported in part by Department of Energy contract DE-AC02-76SF00515. 


\section{Introduction}

$C P$ violation has been established in processes involving $B^{0}-\bar{B}^{0}$ oscillations through measurements of the time dependence of neutral $B$-meson decays to final states which include charmonium [1]. Direct $C P$ violation occurs when the amplitude for a process $i \rightarrow f$ is different from the $C P$ conjugate one: $\bar{i} \rightarrow \bar{f}$. In the Standard Model (SM) this can occur as a result of interference among at least two contributing amplitudes which carry different weak and strong phases. For a decay process $B \rightarrow f$ and its $C P$ conjugate $\bar{B} \rightarrow \bar{f}$ the direct $C P$ asymmetry is defined as:

$$
\mathcal{A}_{C P}=\frac{\Gamma(\bar{B} \rightarrow \bar{f})-\Gamma(B \rightarrow f)}{\Gamma(\bar{B} \rightarrow \bar{f})+\Gamma(B \rightarrow f)}
$$

where $B$ can be either a charged or neutral $B$ meson. If $A_{1}$ and $A_{2}$ are two of the contributing amplitudes to the decay, which have $\Delta \phi_{w}$ weak phase difference and $\Delta \phi_{s}$ strong phase difference, the direct $C P$ asymmetry can be expressed by:

$$
\mathcal{A}_{C P}=\frac{2 \sin \Delta \phi_{w} \sin \Delta \phi_{s}}{\left|A_{1} / A_{2}\right|+\left|A_{2} / A_{1}\right|+2 \cos \Delta \phi_{w} \Delta \phi_{s}} .
$$

This implies that large direct $C P$ violation is expected when the contributing amplitudes have large $\Delta \phi_{s}, \Delta \phi_{w}$ and similar magnitudes. This happens in charmless $B$ decays where there are often contributions from Cabibbo suppressed tree amplitudes as well as from penguin diagrams which have different phases but similar magnitudes. The best candidate in this sense is $B^{0} \rightarrow K^{+} \pi^{-}$ decay.

The simplest approach to the direct $C P$ violation measurement is to measure time integrated asymmetries, for example different rates for the process $B^{+} \rightarrow f^{+}$versus $B^{-} \rightarrow f^{-}$, or in self tagging neutral $B$ decays, for which the final state particles indicates the flavour of the $B$.

Direct $C P$ violation can also be observed as a time dependent asymmetry. In neutral $B$ decays to a $C P$ eigenstate the time dependent asymmetry can be expressed by:

$$
\mathcal{A}_{C P}(\Delta t)=S \sin \left(\Delta m_{d} \Delta t\right)+C \cos \left(\Delta m_{d} \Delta t\right)
$$

where $\Delta m_{d}$ is the $B^{0}-\bar{B}^{0}$ mixing frequency and $\Delta t$ is the difference between the signal and tag $B$ decay times. If the parameter $C$ is different from zero this is an indication of direct $C P$ violation. In this case we define the direct $C P$ violation $\mathcal{A}_{C P}=-C$.

$C P$ violation in charmless $B$ decays can be also interpreted in terms of parameters of the Unitarity Triangle which describes the unitarity of the Cabibbo-Kobayashi-Maskawa quark mixing matrix [2].

In this note we briefly review some recent measurements of direct $C P$ violation at the $B A B A R$ experiment situated at the PEP-II $e^{+} e^{-}$storage ring at the Stanford Linear Accelerator Center [3]. The analyses presented mostly uses about $316 \mathrm{fb}^{-1}$ of data recorded at the $\Upsilon(4 S)$ resonance.

\section{Basic Measurement Strategy}

Events are fully reconstructed combining tracks and neutral clusters in the detector. In order to select $B$ candidates we use a set of two kinematic variables: the beam-energy-substituted mass $m_{\mathrm{ES}}=\sqrt{\left(s / 2+\mathbf{p}_{i} \cdot \mathbf{p}_{B}\right)^{2} / E_{i}^{2}+p_{B}^{2}}$, and the energy difference $\Delta E=E_{B}^{*}-\sqrt{s} / 2$. Here, $\left(E_{i}, \mathbf{p}_{i}\right)$ is the four-vector of the initial $e^{+} e^{-}$system, $\sqrt{s}$ is the center-of-mass energy, $\mathbf{p}_{B}$ is the reconstructed 
momentum of the $B^{0}$ candidate, and $E_{B}^{*}$ is its energy calculated in the $e^{+} e^{-}$rest frame. For signal decays, the $m_{\mathrm{ES}}$ distribution peaks near the $B^{0}$ mass with a resolution of about $2.5 \mathrm{MeV} / c^{2}$, and the $\Delta E$ distribution peaks near zero with a resolution of $10-50 \mathrm{MeV}$, depending on the final state. $\Delta E$ depends on the mass hypothesis assigned to the tracks and is so used in final states with kaons or pions. Particle identification (PID) also uses the the Cherenkov detector (DIRC), which gives excellent $K / \pi$ separation $(>2.5 \sigma$ for momenta $<4.0 \mathrm{GeV} / c)$.

The main background comes from light quark continuum production, which is different by signal in the more jet-like distribution of the decay products. Different variables, as the Legendre polynomials, combined using algorithms which maximize the separation between signal and background, are used to reject most of this background. Another source of background comes from decays of $B$ mesons which mimic the signal. This background is typically more difficult to suppress.

The analysis strategy, common to the most of the analyses, is to perform an unbinned maximum likelihood (ML) fit to several selection variables to extract $C P$ asymmetries simultaneously to the signal yields. Large sidebands, i.e. regions where the signal contribution is negligible, are kept in the selection in order to characterize the background directly on data. Background from other $B$ decays is usually estimated from Monte Carlo samples.

\section{Results on direct $C P$ Violation}

\section{$3.1 \quad B^{0} \rightarrow K^{+} \pi^{-}, B^{0} \rightarrow \pi^{+} \pi^{-}$}

In $B^{0} \rightarrow K^{+} \pi^{-}$decay the charge of the kaon can be used to infer the tag of the $B$ meson, so a time integrated $C P$ violation measurement is feasible. The analysis reconstructs simultaneously $B^{0} \rightarrow h^{+} h^{-}$, with $h=K, \pi$, making use of $m_{\mathrm{ES}}, \Delta E$, a Fisher discriminant $\mathcal{F}$ with event shape variables [4] and the Cherenkov angle $\theta_{c} . \Delta E$ and $\theta_{c}$ lead to the separation of the $h^{+} h^{-}$modes. The $\theta_{c}$ PDFs are determined separately for positive and negative tracks from dedicated $D^{*+} \rightarrow D^{0} \pi^{+}$, $D^{0} \rightarrow K^{-} \pi^{+}$data control samples. The fit to 347 million $B \bar{B}$ pairs returns $2542 \pm 67 B^{0} \rightarrow K \pi$ events, and an asymmetry $\mathcal{A}_{K \pi}=-0.108 \pm 0.024 \pm 0.008$. As a cross-check, the measured $C P$ asymmetry for the background is consistent with zero. Fig. 11 shows the background-subtracted $\Delta E$ distribution for $K^{+} \pi^{-}$events and $K^{-} \pi^{+}$events. The significance of the $C P$ violation, including systematics, is $4.3 \sigma[5]$.

The $C P$ asymmetry for $B^{0} \rightarrow \pi^{+} \pi^{-}$is measured with a time-dependent fit to the $B^{0} \rightarrow h^{+} h^{-}$ sample. The fit yields $675 \pm 42$ signal events, with a direct $C P$ asymmetry parameter $\mathcal{A}_{C P}=-C=$ $0.16 \pm 0.11 \pm 0.03$, consistent with zero.

\section{$3.2 \quad B^{+} \rightarrow h^{+} \pi^{0}$}

In the SM the charge asymmetry in $B^{+} \rightarrow K^{+} \pi^{0}$ is expected to be of the same order of the $B^{0} \rightarrow K^{+} \pi^{-}$one, while no asymmetry is expected in $B^{+} \rightarrow \pi^{+} \pi^{0}$, since the decay is mediated by only one amplitude. The main $B$ backgrounds for these modes are $B \rightarrow \rho \pi, B \rightarrow \rho K$ and $B \rightarrow K^{*} \pi$ decays. The fit strategy is similar to the one of $B^{0} \rightarrow K^{+} \pi^{-}$. The measured yields, branching fractions and $C P$ asymmetries are shown in Table 1 The asymmetries are consistent with zero [5]. 


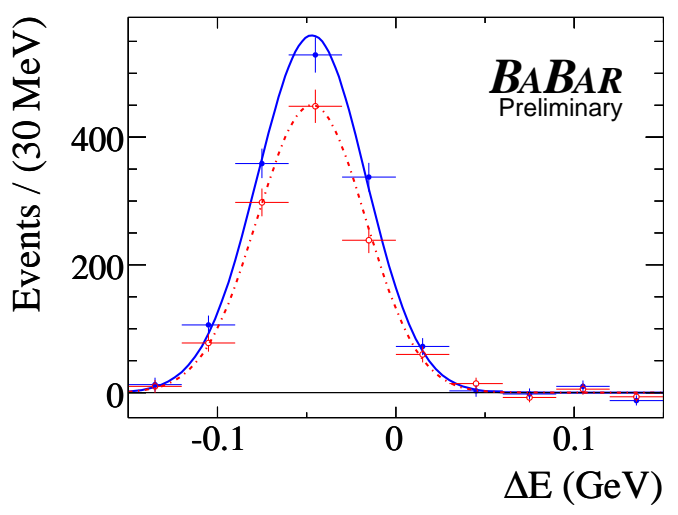

Figure 1: The background-subtracted distribution of $\Delta E$ for signal $K^{ \pm} \pi^{\mp}$ events, comparing $B^{0}$ (solid) and $\bar{B}^{0}$ decays (dashed).

\begin{tabular}{lcc}
\hline \hline & $B^{+} \rightarrow \pi^{+} \pi^{0}$ & $B^{+} \rightarrow K^{+} \pi^{0}$ \\
\hline$N_{\mathrm{S}}$ & $572 \pm 53$ & $1239 \pm 52$ \\
$\mathcal{B}\left(10^{-6}\right)$ & $5.12 \pm 0.47 \pm 0.29$ & $13.3 \pm 0.56 \pm 0.64$ \\
$\mathcal{A}_{C P}$ & $-0.02 \pm 0.09 \pm 0.01$ & $0.02 \pm 0.04 \pm 0.01$ \\
\hline \hline
\end{tabular}

Table 1: Signal yields $\left(N_{\mathrm{S}}\right)$, branching fractions $(\mathcal{B})$ and $C P$ asymmetries for $B^{+} \rightarrow h^{+} \pi^{0}$.

\section{$3.3 \quad B^{+} \rightarrow \phi K^{+}, B \rightarrow \phi \pi$}

The $B^{+} \rightarrow \phi K^{+}$and $B^{ \pm, 0} \rightarrow \phi \pi^{ \pm, 0}$ in the SM proceed only through $b \rightarrow s$ and $b \rightarrow d$ gluonic penguins, respectively, then the direct $C P$ asymmetry is expected to be zero. However, these decays are sensitive to new physics entering the loop amplitude and providing additional phases, which could provide large $C P$ asymmetries and a $\mathcal{B}(B \rightarrow \phi \pi)>10^{-8}$. The analysis uses an extended ML fit to kinematic and event shape variables, the helicity of the $\phi$ meson and $\theta_{c}$. The $\phi$ meson is defined as the $K^{+} K^{-}$pair with an invariant mass in $1.0045<m_{K^{+} K^{-}}<1.0345 \mathrm{GeV} / c^{2}$. The extracted yield for $B^{+} \rightarrow \phi K^{+}$in 347 million $B \bar{B}$ pairs is $624 \pm 30$ events, with a direct $C P$ asymmetry $\mathcal{A}_{C P}=0.046 \pm 0.046 \pm 0.017$, which is consistent with zero [6].

A fit to 232 million $B \bar{B}$ pair returns no signal events for $B \rightarrow \phi \pi$ decays, allowing to set upper limits on $\mathcal{B}$ 's: $\mathcal{B}\left(B^{+} \rightarrow \phi \pi^{+}\right)<2.4 \times 10^{-6}$ and $\mathcal{B}\left(B^{0} \rightarrow \phi \pi^{0}\right)<2.8 \times 10^{-6}$ [7].

\section{$3.4 \quad B^{+} \rightarrow K^{0} h^{+}, B^{0} \rightarrow \bar{K}^{0} K^{0}$}

In SM, decays $B^{+} \rightarrow \bar{K}^{0} h^{+}$and $B^{0} \rightarrow K^{0} \bar{K}^{0}$ proceed only through penguin transition $b \rightarrow d$, then the direct $C P$ asymmetry is expected to vanish. In a way analogous to $B^{+} \rightarrow \phi K^{+}$, new physics effects could enhance it. The direct $C P$ asymmetry in the neutral mode is measured with a time-dependent fit. From an extended ML fit to $m_{\mathrm{ES}}, \Delta E$ and $\mathcal{F}$ (including $\Delta t$ for $B^{0} \rightarrow \bar{K}^{0} K^{0}$ ), in a sample of 347 million $B \bar{B}$ pairs, the signal yields and the $C P$ asymmetries are extracted. The results are reported in Table 2. We observe for the first time the decays $B^{+} \rightarrow \bar{K}^{0} K^{+}$and $B^{0} \rightarrow \bar{K}^{0} K^{0}$ with significances of $5.3 \sigma$ and $7.3 \sigma$, respectively. The measured direct $C P$ asymmetries are consistent with zero [8], in agreement with theoretical expectations. In Fig. 2] the asymmetry 


\begin{tabular}{lcc}
\hline \hline Mode & $\mathcal{B}\left(10^{-6}\right)$ & $\mathcal{A}_{C P}$ \\
\hline$B^{+} \rightarrow K^{0} \pi^{+}$ & $23.9 \pm 1.1 \pm 1.0$ & $-0.03 \pm 0.04 \pm 0.01$ \\
$B^{+} \rightarrow \bar{K}^{0} K^{+}$ & $1.6 \pm 0.4 \pm 0.1$ & $0.10 \pm 0.26 \pm 0.03$ \\
$B^{0} \rightarrow K^{0} \bar{K}^{0}$ & $1.1 \pm 0.3 \pm 0.1$ & $0.40 \pm 0.41 \pm 0.06$ \\
\hline \hline
\end{tabular}

Table 2: Signal yields $\left(N_{\mathrm{S}}\right)$, branching fractions $\mathcal{B}\left(\times 10^{-6}\right)$ and $C P$ asymmetries for $B^{+} \rightarrow K^{0} h^{+}$, $B^{+} \rightarrow K^{0} \bar{K}^{0}$.

for $K_{S}^{0} K_{S}^{0}$ events and the confidence level contours in the $C$ vs. $S$ plane are shown.
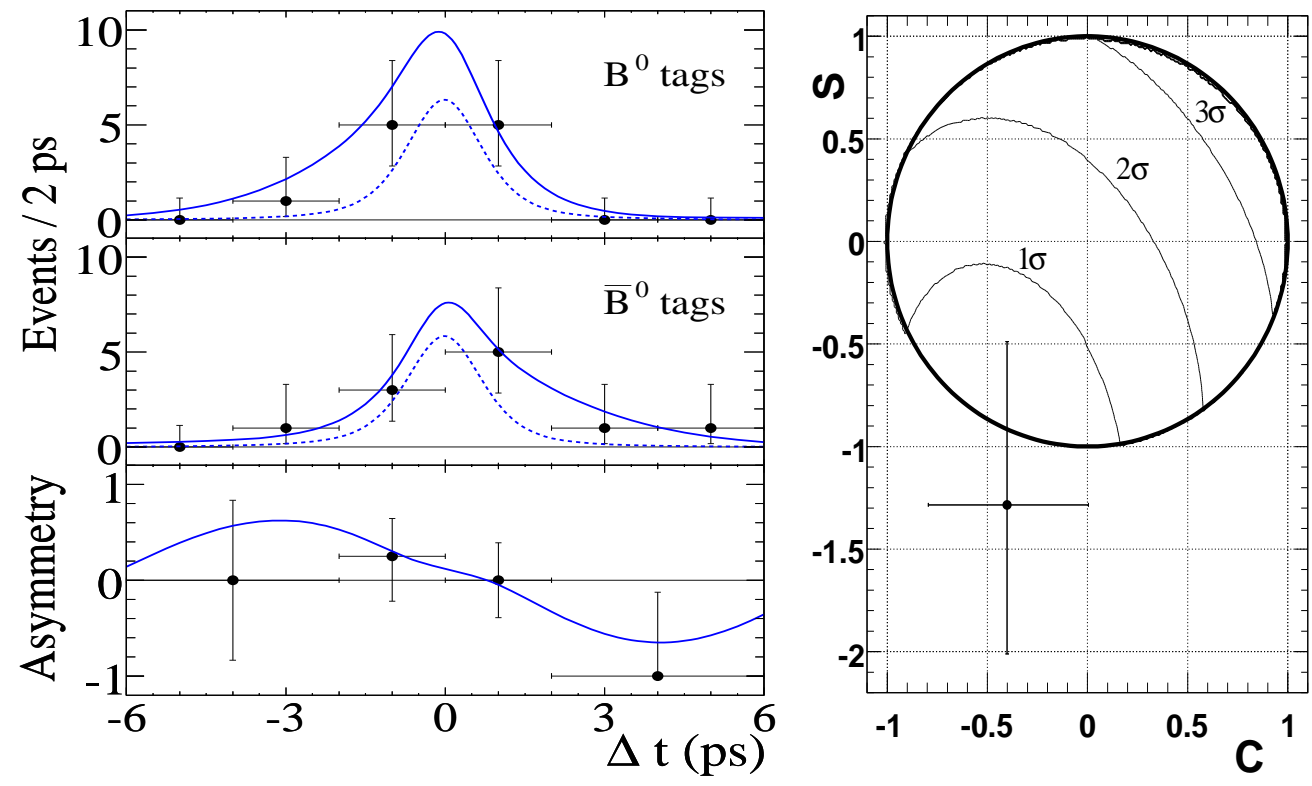

Figure 2: Left: $\Delta t$ distributions for $B^{0} \rightarrow K_{S}^{0} K_{S}^{0}$ decays in data tagged as $B^{0}$ (top) or $\bar{B}^{0}$ (middle), and the asymmetry (bottom). The solid (dotted) curve represents the total (background only) likelihood projection. Right: Confidence level contours in the $C$ vs. $S$ plane. The circle indicates the physically allowed region, while the point with errors is the result on data.

\section{$3.5 \quad B^{0} \rightarrow(\rho \pi)^{0}$}

A time-dependent analysis of the Dalitz plot of the $B^{0} \rightarrow \pi^{+} \pi^{-} \pi^{0}$ decays is performed, in order to measure the $C P$ violation taking into account the interference between the intermediate states: $B^{0} \rightarrow \rho^{ \pm} \pi^{\mp}$ and the color suppressed $B^{0} \rightarrow \rho^{0} \pi^{0}$. QCD factorization predicts null direct $C P$ violation, due to the lack of penguin contributions, but non-factorizable effects could be present, modifying this conclusion. Through the interference between these amplitudes this measurement can lead to an unambiguous determination of the angle $\alpha$ of the Unitarity Triangle 9 . An extended ML fit to $m_{\mathrm{ES}}, \Delta E, \mathcal{F}$, Dalitz plot variables and $\Delta t$ is performed to 347 million $B \bar{B}$ pairs. Since the final states are not $C P$ eigenstates the $C P$ violation can be expressed in terms of the time-dependent 
$C$ parameter and time- and flavour-integrated $C P$ asymmetry $\mathcal{A}_{\rho \pi}$. These can be rearranged in terms of $\mathcal{A}_{\rho \pi}^{+-}$which describes the direct $C P$ violation of the amplitude where the $\rho$ is emitted from the $W$, and $\mathcal{A}_{\rho \pi}^{-+}$which describes amplitudes where the $\pi$ is emitted from the $W$. The fit returns a signal yield of $1847 \pm 69$ events, dominated by $B^{0} \rightarrow \rho^{ \pm} \pi^{\mp}$ decays, and direct $C P$ asymmetries $\mathcal{A}_{\rho \pi}^{+-}=0.03 \pm 0.07 \pm 0.03, \mathcal{A}_{\rho \pi}^{-+}=-0.38_{-0.16}^{+0.15} \pm 0.07$. These results show an evidence of direct $C P$ violation, including systematics, of $3.0 \sigma$ [10]. Fig. 3] shows confidence level contours for $\mathcal{A}_{\rho \pi}^{+-}$and $\mathcal{A}_{\rho \pi}^{-+}$.

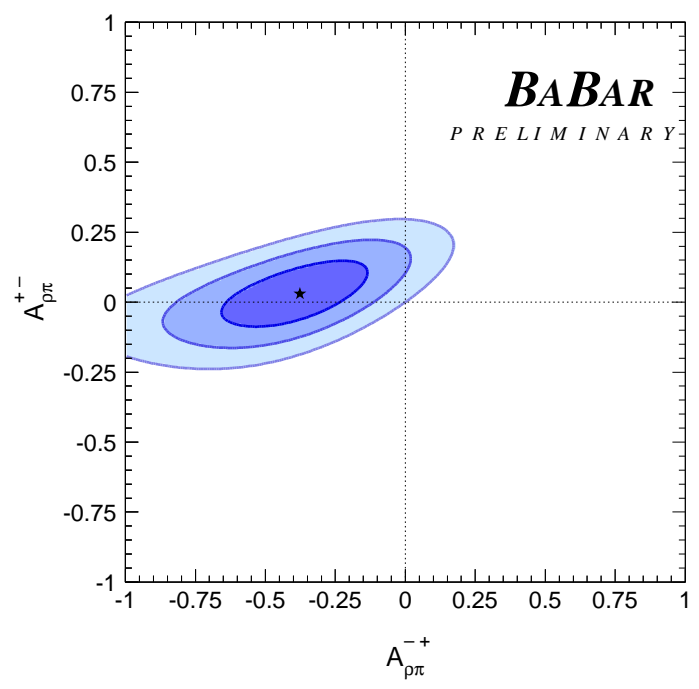

Figure 3: Confidence level contours for the direct $C P$ asymmetries $\mathcal{A}_{\rho \pi}^{+-}$and $\mathcal{A}_{\rho \pi}^{-+}$. The shaded areas represent $1 \sigma, 2 \sigma$ and $3 \sigma$ contours, from darker to lighter.

\section{Conclusions}

The direct $C P$ violation has been measured in $B^{0} \rightarrow K^{+} \pi^{-}$decays to be of $0.108 \pm 0.025$, resulting in a $4.3 \sigma$ effect. We observed for the first time the decays $B^{+} \rightarrow \bar{K}^{0} K^{+}$and $B^{0} \rightarrow K^{0} \bar{K}^{0}$, with no $C P$ violation. The direct $C P$ asymmetry is consistent with zero in other rare decay modes, consistently with the SM predictions for those decays which are mostly sensitive to new physics effects, as $B^{+} \rightarrow \phi K^{+}$. In the time-dependent analysis of $B^{0} \rightarrow(\rho \pi)^{0}$ we see an evidence of direct $C P$ violation with $3.0 \sigma$ significance. The increasing of the recorded luminosity in the next years will provide a powerful tool to investigate the SM picture of the nature of $C P$ violation.

\section{Acknowledgements}

I am grateful to my BABAR collegues Gianluca Cavoto, Maurizio Pierini, Denis Dujmic, and Mahalaxmi Krishnamurthy for their support in this work. I would like to thank also Daniele del Re and prof. Aaron Roodman for their support during the conference. 


\section{References}

[1] B. Aubert et al. [BABAR Collaboration], arXiv:hep-ex/0607107, K. Abe et al. [Belle Collaboration], arXiv:hep-ex/0608039.

[2] UTfit Collaboration, M. Bona et al., hep-ph/0501199. CKMfitter Group, J. Charles et al., Eur. Phys. J. C 41, 1 (2005)

[3] B. Aubert et al. [BABAR Collaboration], Nucl. Instrum. Meth. A 479, 1 (2002) arXiv:hep-ex/0105044.

[4] R. A. Fisher, Annals of Eugenics 7, 179 (1936).

[5] B. Aubert et al. [BABAR Collaboration], arXiv:hep-ex/0607106.

[6] B. Aubert et al. [BABAR Collaboration], arXiv:hep-ex/0607112.

[7] B. Aubert et al. [BABAR Collaboration], Phys. Rev. D 74, 011102 (2006) arXiv:hep-ex/0605037.

[8] B. Aubert et al. [BABAR Collaboration], arXiv:hep-ex/0608036.

[9] A. E. Snyder and H. R. Quinn, Phys. Rev. D 48, 2139 (1993).

[10] B. Aubert [BABAR Collaboration], arXiv:hep-ex/0608002. 